\title{
Graphic analysis of pulmonary mechanics in neonates receiving assisted ventilation
}

\author{
Sunil K Sinha, Joanne J Nicks, Steven M Donn
}

Division of Women and Children, Directorate of Neonatology, South Cleveland Hospital, Middlesbrough Cleveland TS4 3BW S Sinha

Department of Pediatric Respiratory Care

J Nicks

\section{Department of} Pediatrics (Division of Neonatal-Perinatal Medicine),

University of Michigan Medical Center, Ann Arbor, Michigan, USA SMA Donn

Correspondence to: Dr Sunil Sinha.
With the increasing complexity of neonatal respiratory care, pulmonary mechanics testing is emerging as a valuable tool to aid clinical decision making in the management of ventilated infants. Although there are as yet no published randomised controlled clinical trials to suggest that pulmonary mechanics testing reduces mortality or morbidity, it has-in conjunction with clinical, radiological, and blood gas monitoring - changed neonatal ventilation from "good judgment" to "informed judgment." It is not surprising that pulmonary mechanics testing is increasingly becoming an essential element in the assessment of patient status, therapeutic evaluation, and management guidance of infants with ventilator dependence. A working knowledge of pulmonary mechanics also improves understanding of pulmonary physiology and pathophysiology and their responses to mechanical ventilatory support. ${ }^{2}$ Unfortunately, these graphical data continue to be additional information that the average bedside clinician is currently unsure how to interpret.

The purpose of this article is to disseminate the information on this technique based on our experience using the Bird Graphic Monitor (Bird Products Corporation, Palm Springs, California, USA), which in conjunction with its Partner IIi Monitor and VIP Bird Infant/ Pediatric Ventilator, provides a continuous display of graphic representation of pressure, flow, or volume waveforms, or relations between pressure-volume and flow-volume in the form of loops. It also gives numerical values for pressure, tidal volume, and flow, and can be used for establishing trends up to 24 hours. All the above graphics can be obtained as printouts when used with a standard computer printer.

\section{Background}

Pulmonary mechanics monitoring consists of measurements of several variables which define different aspects of lung function. Although it has become popular to refer to this type of analysis as "pulmonary function testing," measurements of functional residual capacity (FRC) are not included. Devices are becoming available to determine FRC at the bedside, using either nitrogen washout or helium dilution techniques, but at present the methodology is extremely expensive and impractical. Many different pulmonary mechan- ics tests can be performed on ventilated infants; only a few are commonly used in clinical practice. Specifically, clinicians are interested in the pressure necessary to cause a flow of gas to enter the airway and increase the volume of the lungs. From these variables, several other measures of pulmonary mechanics can also be derived, such as pulmonary compliance and resistance, and resistive work of breathing (energetics). Compliance is the term used to describe the relation between a change in volume and the pressure required to produce that change: $\mathrm{C}=\Delta \mathrm{V} / \mathrm{P}$. It gives information about the elasticity of the lungs. Resistance is a result of friction of gas flow against the air conducting system. It is roughly measured as the change in transpulmonary pressure divided by change in air flow, and is an indicator of airway function. The product of compliance and resistance determines the time constant, which is a measure of how quickly the lungs can inhale or exhale, or how long it takes for the alveoli and proximal airway pressure to equilibrate. From these mechanics, which can be displayed as numerical values or as graphic signals, useful information can be obtained and used for diagnosing specific lung pathology, evaluating disease progression, and determining therapeutic interventions.

Although the facilities to monitor airway pressure, tidal volume, and gas flow in ventilated infants have existed for some time, their application has been mostly limited to research purposes. However, recent advances in microprocessor technology for on-line analysis of pulmonary mechanics have made such evaluations easily available for bedside clinical application. ${ }^{134}$ Indeed, most of the new ventilators either come with, or have an option for, a graphical display, and this has become an essential feature of the newer ventilators available for infant/paediatric use. These on-line systems obviate the need for interrupting ventilation. In addition, because of technological advantages, the sensors are very light in weight and add minimal dead space to the ventilatory circuit. This permits their application to even the smallest preterm infants.

\section{Putative advantages}

The rationale of pulmonary mechanics testing in ventilated infants is based on the assumption that early identification of pulmonary prob- 

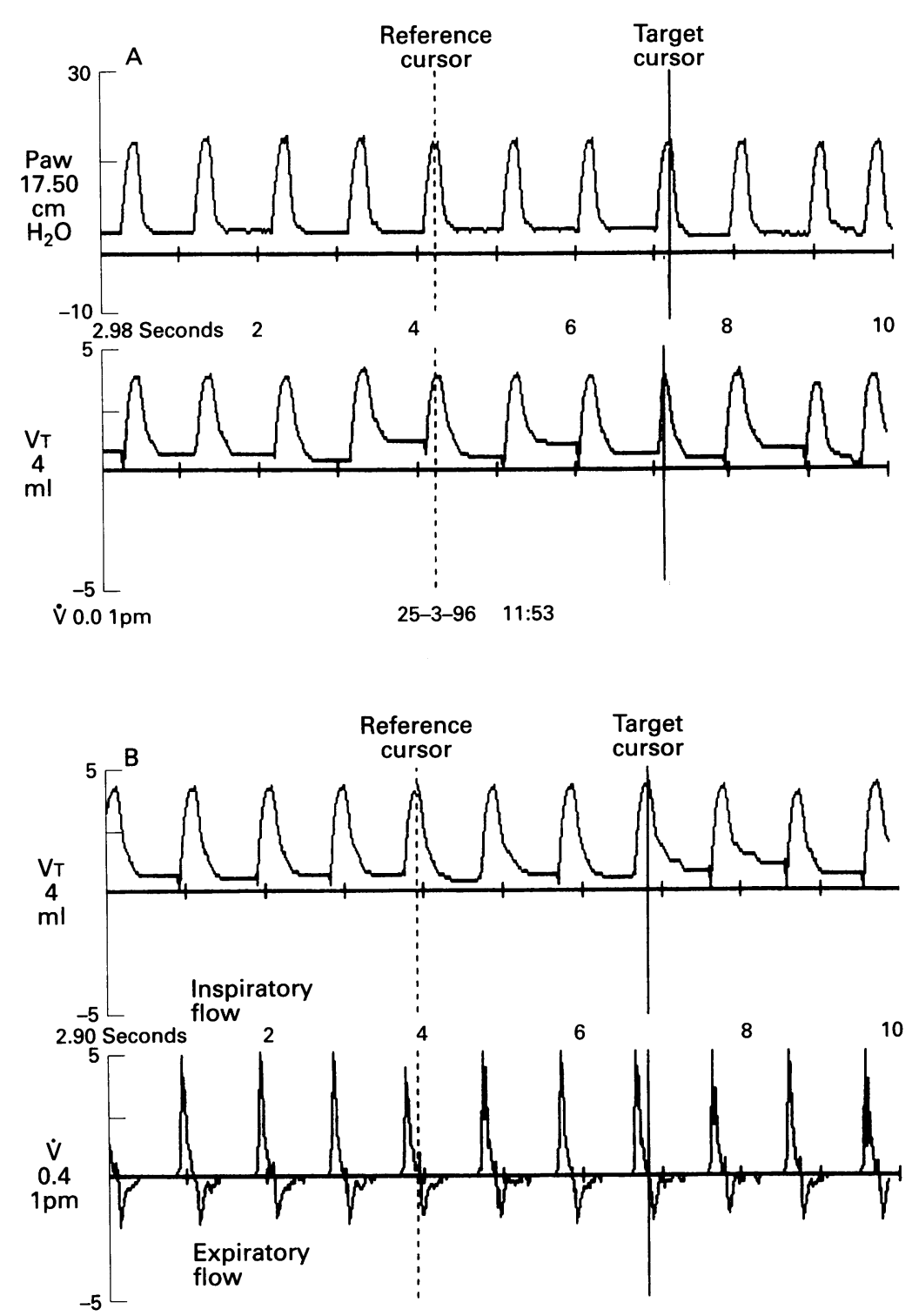

Paw $19.00 \mathrm{~cm} \mathrm{H}_{2} \mathrm{O}$

25-3-96 11:44

Figure 1 Scalar waveforms for airway pressure (PAW) and tidal volume $(V T)(A)$, and tidal volume and airway flow $(V)(B)$. Time intervals can be measured using a reference cursor (dotted line). A target cursor can be moved to any given point on a wave to obtain numerical values of pressure, volume, or flow at that point.

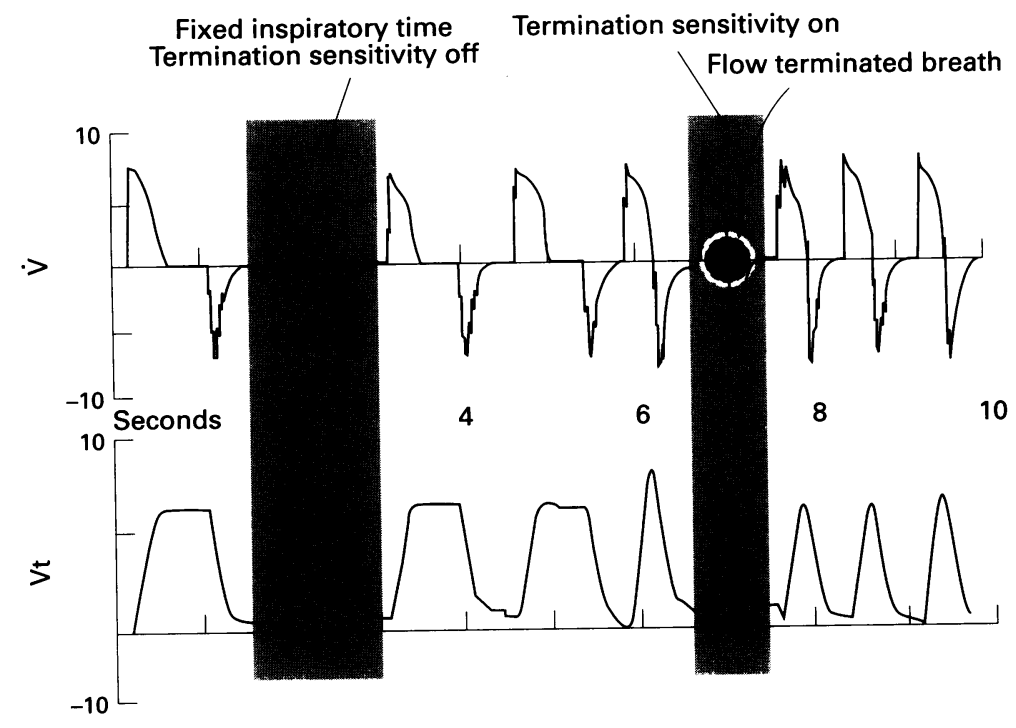

Figure 2 Flow and volume waveforms showing an initial inspiratory time which is too long, but which is subsequently shortened by use of termination sensitivity mode. lems, either inherent or iatrogenic, and institution of appropriate therapeutic or ventilatory adjustments will improve the dysfunction and/or reduce the incidence of acute and chronic lung injuries. ${ }^{56}$ Besides assessment of acute respiratory distress and evaluation of mechanical ventilation, potential benefits of on-line pulmonary graphics include assessment of suitability for weaning, monitoring of complex treatments such as extracorporeal membrane oxygen (ECMO) or nitric oxide, and follow up of chronic lung disease.

\section{Methods}

The two forms of respiratory graphics most widely utilised in clinical practice are scalar waveforms and loops, both showing simultaneous relations between pressure, volume, and air flow. Although the software program which monitors and plots these graphs can also give numerical values for each variable and calculated parameter, in practice, most of the useful information can be derived by the visual inspection of their morphology. This varies, according to specific respiratory function status, provided allowance has been made for errors arising from inaccurate calibration and artefacts.

It is beyond the scope of this paper to detail the specific methods through which the basic signals of flow, volume, and pressure undergo transduction to graphic or numeric displays, and several references are available for such purpose. ${ }^{78}$ However, the same microprocessor based technology used to provide patient triggered ventilation is used in conjunction with highly sensitive transducers generally placed close to the endotracheal tube connector.

\section{Respiratory waveforms}

Figure 1 shows a simultaneous representation of pressure and volume (A) and volume and flow (B) scalar waveforms during trigger ventilation in time cycled pressure limited mode. The magnitude of individual parameters and their time interval can be measured by use of the reference and target cursors. The basic interpretation and the clinical application of these waveforms are described below.

\section{FLOW WAVEFORMS}

Flow may be thought of as the volume of gas delivered per unit of time. Inspiratory flow is plotted above the abscissa (positive) and expiratory flow is plotted below the abscissa (negative). The intersection of the abscissa and ordinate occurs at zero flow. The duration and shape of the inspiratory and expiratory flow waveform are affected by many factors, including type of ventilation, inspiratory:expiratory time ratio, impedance to gas flow during expiration, and effect of therapeutics, such as bronchodilators.

The major advantage of looking at flow waveforms is in assessing the amount of flow that occurs during the inspiratory phase to see if the set inspiratory time is inappropriately long. Figure 2 shows a flow waveform with fixed inspiratory time. This may be needed to achieve a high mean airway pressure and better 

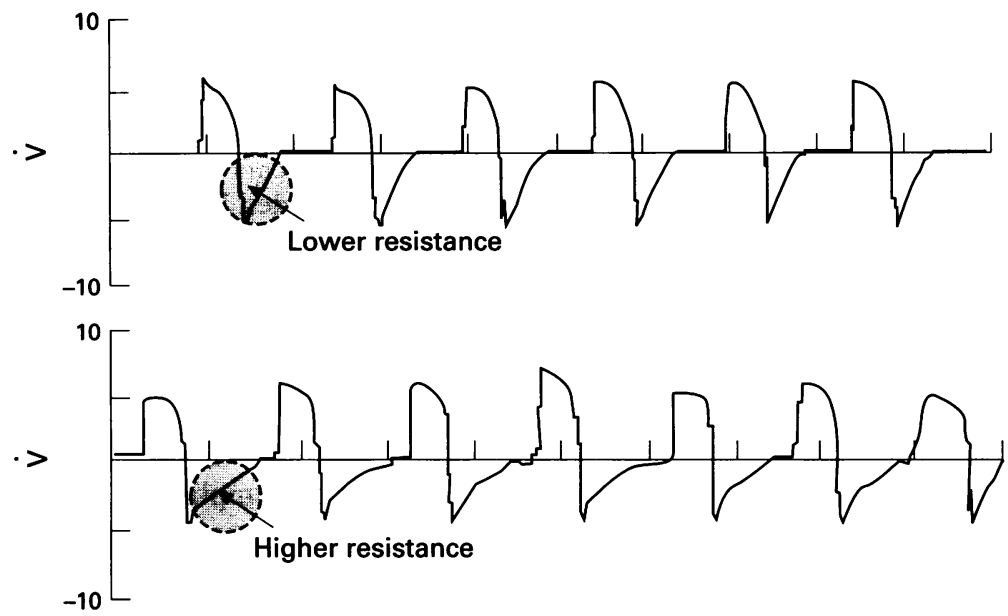

Figure 3 Flow waveforms. Upper tracing shows lower expiratory resistance. Increased expiratory resistance in bottom tracing results in flattened slope and slow return to baseline.

oxygenation during the acute stages of respiratory distress syndrome with stiff lungs and short time constants, but once the lungs become compliant and if baby is breathing at a fast rate especially while on patient trigger ventilation mode, fixed inspiratory time may prove to be inappropriately long. This is likely (mathematically) to result in insufficient expiratory time and contribute towards air trapping. In these situations, inspiratory flow can be limited by either shortening the inspiratory time or instituting a mode called "termination sensitivity" (in VIP Bird Ventilators), which limit the inspiratory flow to a preset value between $75-95 \%$ of peak inspiratory flow and then trigger the expiratory phase. This permits complete synchronisation. The duration and shape of expiratory flow waveforms depend both on

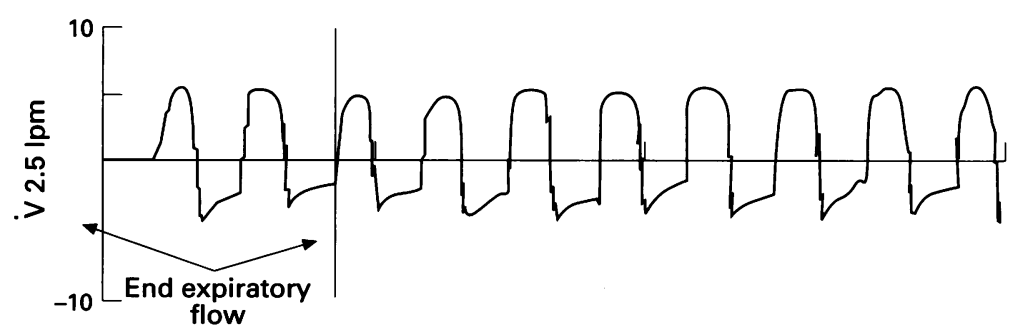

Figure 4 Flow waveform shows air trapping. Expiratory flow (below baseline) never reaches zero before next breath is initiated.

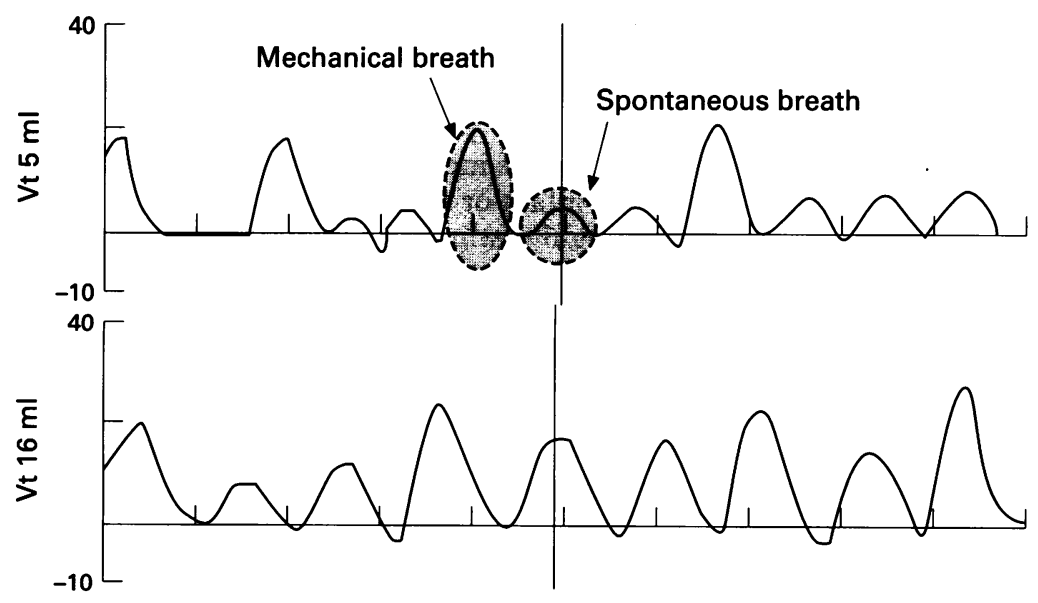

Figure 5 Volume waveform. Top tracing shows ineffective spontaneous breaths; lower tracing shows improved tidal volume delivery during spontaneous breathing. the resistance and the compliance, but because of the small diameter endotracheal tubes used in neonates, resistance is a more important determinant for its configuration. The expiratory waveform can be used to observe changes in expiratory resistance (fig 3 ) and the presence of gas trapping (fig 4). Normally, expiratory flow reaches a zero flow state before the next breath begins. If the expiratory time is too short, the expiratory flow fails to reach a zero flow state before the next breath starts, thus suggesting that gas trapping may be occurring. A similar appearance as shown in fig 4 can happen due to a large leak around the endotracheal tube, but this can be differentiated either by using the pressure-volume loops which fail to close, or by monitoring the difference between inspiratory and exhaled tidal volume.

VOLUME AND PRESSURE WAVEFORMS

Measurement of tidal volume is becoming increasingly important in ventilatory management. The desired inspiratory tidal volume for a ventilated breath ranges between 5.2-7.2 $\mathrm{ml} / \mathrm{kg}^{9}$ while a tidal volume of $3.5-5 \mathrm{ml} / \mathrm{kg}$ for spontaneous breaths generally indicates suitability of the infant for weaning, provided this is matched by satisfactory arterial blood gases which remain the best index of ventilatory adequacy. Minute ventilation (tidal volume $\mathrm{x}$ respiratory rate) is also currently being evaluated as one of the predictor of weaning $(250-400 \mathrm{ml} / \mathrm{kg} / \mathrm{minute}),{ }^{10}$ especially in smal infants who have short inspiratory times and a higher respiratory frequency. Unlike flow waveforms, both the inspiratory and expiratory phase of volume and pressure waveforms are positive and are therefore plotted above the abscissa. Breath-to-breath variability and longer term trends are useful in selecting the mode of ventilation and individualised adjustments to customise ventilator parameters for each patient. Figure 5 shows differences in tidal volume delivery between mechanical and spontaneous breaths during synchronised intermittent mandatory ventilation (SIMV). The top waveform indicates that spontaneous tidal volumes inbetween mechanically delivered breaths are ineffective. On the bottom graph, the infant has much higher spontaneous tidal volumes suggesting readiness to wean.

\section{Pulmonary mechanics loops}

The loops are commonly used to demonstrate correlations between airway pressure and volume, and airflow and lung volume (fig 6). Recently, attempts have also been made to produce a flow-pressure loop, but it is not yet available in clinical practice. At present, there is no agreed convention regarding the manner in which the loops are generated. Some devices draw them clockwise, others anticlockwise. Some label upward deflections as negative, others positive. The clinician must be familiar with these differences to be able accurately to interpret the presented data.

PRESSURE-VOLUME LOOPS

These loops graphically depict the correlations between ventilator inspiratory pressure and the 

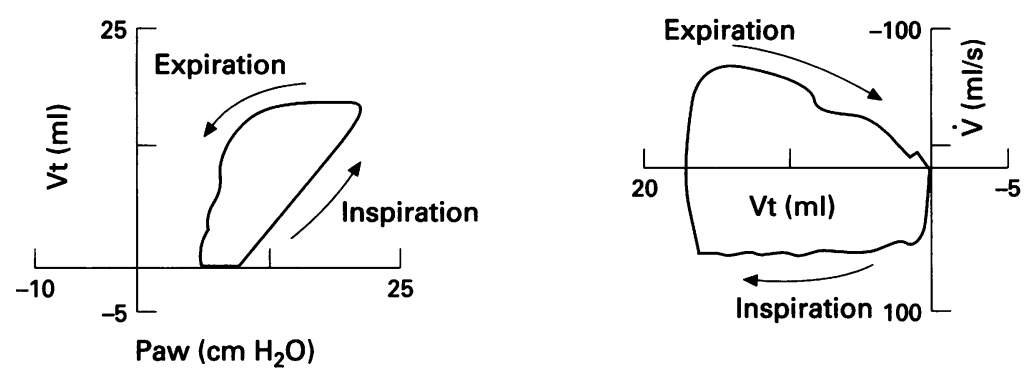

Figure 6 Pulmonary mechanics loops. Left graph displays pressure-volume relations. Note inspiratory and expiratory limbs, drawn counterclockwise on the Bird Graphic Monitor. On the right is the flow-volume relation drawn clockwise, in which airflow to the patient (inspiration) is plotted positively, airflow away from the patient (expiration) is plotted negatively.
Paw $\left(\mathrm{cm} \mathrm{H} \mathrm{H}_{2} \mathrm{O}\right)$

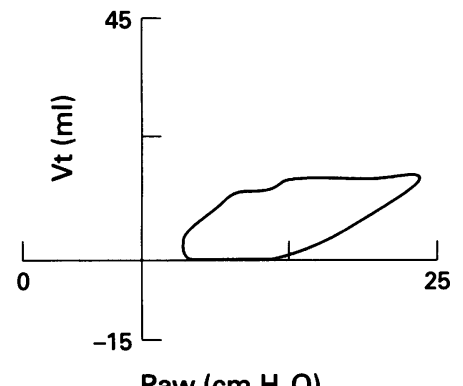

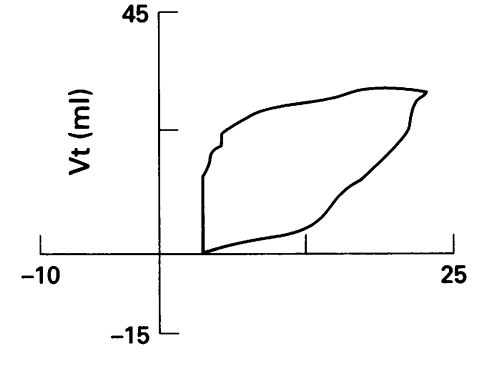

Paw $\left(\mathrm{cm} \mathrm{H}_{2} \mathrm{O}\right)$
Figure 7 Pressure-volume relation. On the left, the compliance line would have an axis about $30^{\circ}$ above horizontal, indicating poor compliance. On the right, the compliance line has improved to about $45^{\circ}$. This scenario might be seen in an infant with respiratory distress syndrome after surfactant administration.
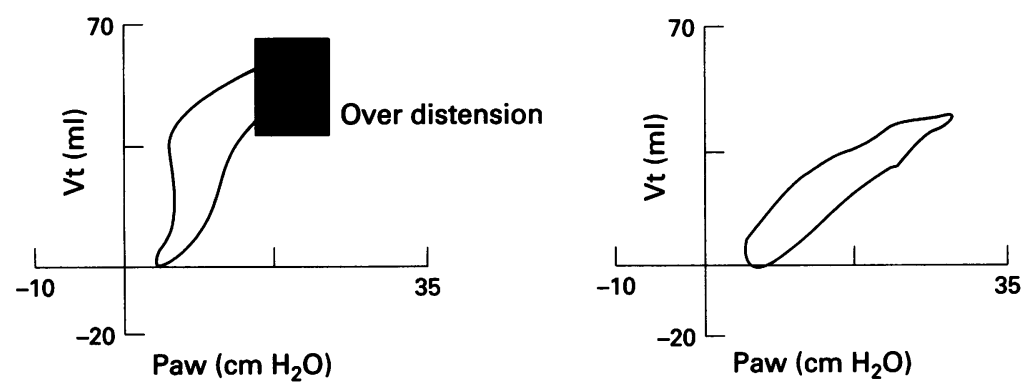

Figure 8 Pressure-volume relation. Graph on left demonstrates overdistension. Note minimal change in volume over the last $20 \%$ of the breath. A reduction in ventilatory pressure (right graph) normalises the loop.
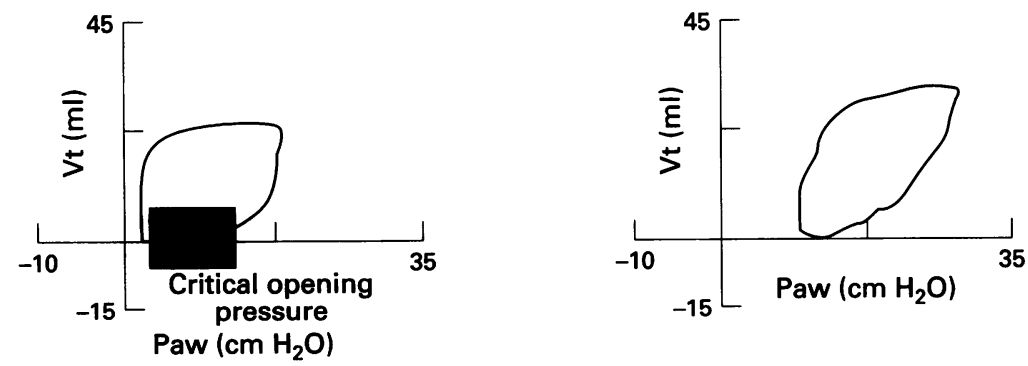

Figure 9 Demonstration of effect of PEEP on pressure-volume relation. Left: inadequate $P E E P$ results in poor volume delivery as pressure increases. Right: higher PEEP results in appropriate volume delivery with increasing pressure.

volume of gas entering the lungs. The horizontal axis represents the degree of positive pressure exerted by the ventilator and the vertical axis represents the gas volume changes in the lungs (fig 7). The lower portion of the loop shows the dynamic relation between volume changes and pressure changes on inspiration, the upper portion of the loop shows volume changes during expiration. A line connecting the points of zero flow-changeover from expiration to inspiration-is the compliance line, and the slope of that line reflects pulmonary compliance. Compliance can be calculated as the change in volume divided by the change in pressure; in practice an estimate of compliance can be made by looking at the slope of the compliance line, which is normally $45^{\circ}$ from the horizontal axis. If this slope is more towards the vertical axis, compliance is improving, and conversely is decreasing if this line moves nearer to the horizontal axis. It must be realised that slope of the compliance line also depends on the calibrations and can be misleading if not adjusted properly. Devices such as VIP Bird have an automatic scaling function which serves to reference the curve such that normal compliance line should be about $45^{\circ}$. Over-distension is implied when little or no change in volume occurs as increasing pressure is delivered. One way to recognise this is to look at the terminal (upper) portion of the pressure-volume loop and see how much tidal volume is being delivered per unit of pressure change. This has been schematically demonstrated in fig 8 created from a test lung for instructive purposes. A useful parameter in this case is the "C20." This can be estimated by calculating total compliance of the lung in relation to the compliance of the last $20 \%$ of the breath (by dividing tidal volume by peak pressure). If the compliance of the last $20 \%$ is less than $80 \%$ of the total compliance, it is suggestive of over-distension, and the ventilator should be adjusted to reach a $\mathrm{C} 20$ value higher than $80 \%$. This may be accomplished by decreasing peak inspiratory pressure, inspiratory time, and in some instances, positive end expiratory pressure.

Pulmonary compliance is a measurement of the distensibility of the lung and is in a sense an indicator of the function of the lung parenchyma. Disease processes that make the lung stiffer and thus decrease pulmonary compliance in neonates include surfactant deficiency states, pneumonia, pulmonary oedema, and pulmonary hypoplasia. Increased resistance, such as that seen in bronchopulmonary dysplasia, causes a "bowing" or hysteresis around the compliance line, and is an indirect measure of the work of breathing (by either the infant or the mechanical ventilator) to move the lungs. This resistive work of breathing increases as pulmonary mechanics worsen, reflecting the increased amount of energy spent by the infant or the ventilator to breathe. It can be roughly estimated by the total area covered by the pressure-volume loop and the area adjacent to its deflation limb and the ordinate. There are, however, major problems in the interpretation of the resistive work of breathing in infants on ventilation, as altering the circuit flow alone can have considerable effects even in the absence of changes in lung mechanics.

The pressure-volume loop may also be used to evaluate optimal peak expiratory end pressure (PEEP) (fig 9). On the left, the loop shows a delay in volume delivery despite increasing pressure, indicating insufficient 

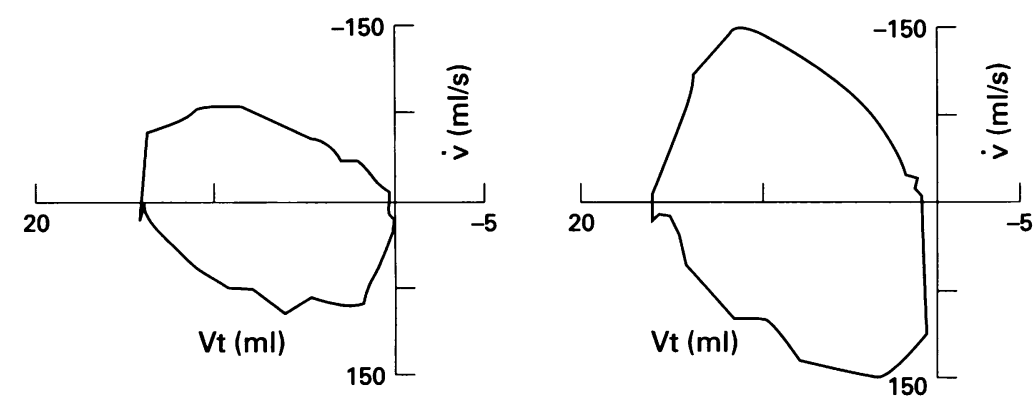

Figure 10 Flow-volume loop. Left graph indicates high resistance, demonstrated by lower flow at a given volume. Right graph indicates lower resistance (such as after bronchodilator treatment) with higher flow at a given volume.
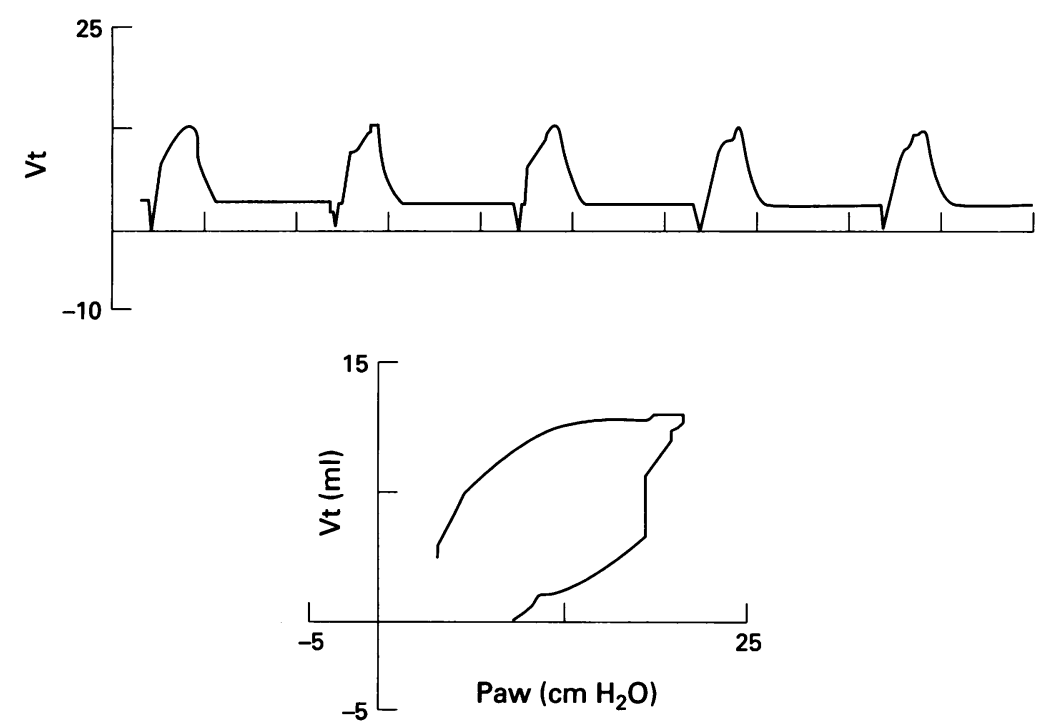

Figure 11 Endotracheal tube leak. Volume waveform fails to reach baseline at end-expiration. Pressure volume loop fails to close.
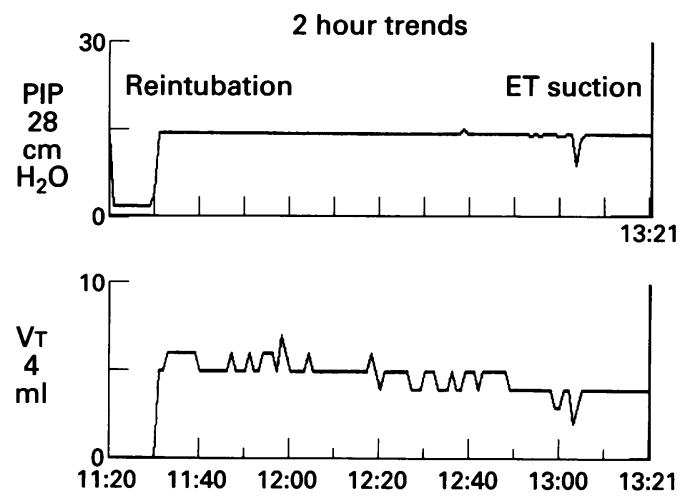

Figure 12 Trend data, pressure-targeted ventilation. Even though peak inspiratory pressure is held constant, tidal volume delivery is variable and decreasing.

opening pressure. An increase in PEEP, shown on the right, results in an immediate rise in volume as pressure is delivered.

FLOW-VOLUME LOOP

Another valuable measurement in the pulmonary mechanics profile is the resistance of gas flow through the airways. This can be assessed by observing the flow-volume loop, which graphically displays the relation between change in volume and change in airflow. High airway resistance results in lower flow for a given volume. Conditions causing high resistance in ventilated babies include airway obstruction from endotracheal tube kinking or blockage with secretions, meconium aspiration syndrome, and bronchopulmonary dysplasia.

Figure 10 shows flow-volume loops, which should be rounded and smooth around the horizontal axis like an egg on its end. The lower portion of the loop represents inspiratory data; the upper limb describes the expiratory correlation. (Because there is no agreed convention on direction of inspiratory and expiratory limb among various manufacturers, it is important to become familiar with the direction of the flow-volume loop on individual machines.) A flow-volume loop depicts changes in inspiratory and expiratory flow against volume changes, and is useful in detecting flow restriction from airway abnormalities. Under conditions of high airway resistance, flow is lower for a given volume. Flow will be lower on inspiration with high inspiratory resistance, or lower on expiration with high expiratory resistance. There is a fixed inspiratory and expiratory resistance associated with an endotracheal tube. The example in fig 10 shows increased inspiratory and expiratory flow from left to right, which might be seen after bronchodilator treatment. This could also happen as a result of high ventilatory pressure or improvement in lung function after an improvement in respiratory distress syndrome. The ability to identify and quantify endotracheal tube leaks may be facilitated through graphic monitoring. Leaks can be identified both on the volume wave form as well as pressure-volume loops (fig 11). Increased resistance and interruption to gas flow from excessive condensation arising in the ventilatory circuit or accumulation of secretions in the airway can be identified by the appearance of jaggedness on graphics.

There are a number of other useful clinical situations, including the assessment of different ventilatory modalities (such as pressure support ventilation) on pulmonary function ${ }^{12}$ and the assessment of patient-ventilator interaction (synchronous versus asynchronous), ${ }^{13}$ There is also an advantage in collecting and evaluating the trends of specific variables such as tidal volume, minute ventilation, and frequency for spontaneous and mechanical breaths as well as peak pressure, inspiratory time, and mean airway pressure. For example, the trend depicted in fig 12 is consistent with an infant on pressure ventilation where despite peak inspiratory pressure (PIP0 remaining constant, tidal volume has gradually decreased. This situation (as in secondary lung complications during respiratory distress syndrome) demands adjustment of ventilatory parameters by the operator. Conversely, tidal volume delivery may automatically increase after improvement in lung compliance despite PIP being the same (as after surfactant replacement), and cause iatrogenic complications if ventilatory adjustments are not made in time.

\section{Limitations}

Despite the major advantages of the current system providing pulmonary mechanics graphics, it should be realised that the information provided about the function of the lungs only complements (and does not substitute) infor 
mation gained by other means of patient monitoring, including clinical signs and blood gas examination. Like radiographs, graphics should only be taken as suggestive of a condition rather than being definitive. Pulmonary graphic waveforms can be misshapen because of inherent inaccuracy of the measurement system (from calibration differences between inspiration and expiration), or from temporary artefacts arising from patient position or impedance to the gas flow by condensation in the ventilatory circuit. It is imperative to correct these pitfalls before accepting the findings of graphics as a guidance to change ventilatory settings or assess the patient's clinical condition. ${ }^{14}$ In this particular respect, the "trends" over a period of time seem to be of more value than individual breath analysis. Although there are few data showing a beneficial or more cost effective approach to neonatal ventilation by the continuous use of pulmonary mechanics monitoring, the development and implementation of this technology should make some studies feasible in the near future.

\section{Conclusion}

On-line pulmonary graphic analysis represents another major advance in respiratory technology which promises to improve the safety and efficacy of neonatal mechanical ventilation. Clinicians are now afforded a breath-to-breath view of pulmonary mechanics and respiratory waveforms. This permits constant surveillance of conditions such as air trapping before they become clinically obvious, and the fine tuning of ventilator settings to customise management according to the problems and responses of the individual patient. Clinicians should avail themselves of this window of opportunity.

We thank Bird Products Corporation for permission to use the figures.

1 MacDonald KD, Wirtschafler DD. Continuous neonatal pulmonary mechanics with the BICORE CP-100 monitor Neo Intensive Care 1992; 5:55-61.

2 MacIntyre NR, Hagus CK. Graphical Analysis of Flow, Pressure and Volume During Mechanical Ventilation. Riverside, CA: Bear Medical Systems, 1987.

3 Goldstein $M$. Continuous in-line pulmonary mechanics. Neo Intensive Care 1993;5:42-5.

4 Lloyd JS, Cvetnic WG. Continuous in-line respiratory monitoring in the critically ill preterm infant. Neo Intensive monitoring in the critic

5 Fisher JB, Mammel MC, Coleman JM, Bing JR, Boros SJ. Identifying lung overdistension during mechanical ventilation by using volume-pressure loops. Pediatr Pulmonol 1988; 5:10-14.

6 Rosen WC, Mammel MC, Bing DR, Boros SJ. Bedside pulmonary function testing reduces pneumothoraces durin infant mechanical ventilation. Pediatr Res 1989; 4:324.

7 Cunningham MD. Monitoring pulmonary function. In Goldsmith JP, Karotkin EH, Barker S, eds. Assisted Ventilation of the Neonate. Philadelphia: WB Saunders Co., 1988: 233-44.

8 Donn SM. Neonatal and Pediatric Pulmonary Graphic Analysis: Principles and Clinical Applications. Armonk, NY: sis: Principles and Clinical

9 Bhutani V, Abbasi S. Evaluation of pulmonary function in the neonate. In: Polin RA, Fox WW, eds. Fetal and Neonathe neonate. In: Polin RA, Fox WW, eds. Fetal and Neom

tal Physiology. Philadelphia: WB Saunders, 1992: 866.
10 Spitzer AR. Mechanical ventilation. In: Spitzer AR,ed. Spitzer AR. Mechanical ventilation. In: Spitzer AR,ed. Mosby Year Book, 1996: 558.

11 Comroe JH, Forster RD, Dubois AB, Briscoe WA, Carlsen E. The Lung: Clinical Physiology and Pulmonary Function Tests. Chicago: Year Book Mecial Publishing, 1962.

12 Nicks J, Becker MA, Donn SM. Bronchopulmonary dysplasia: response to pressure support ventilation. f Perinatol 1994; 14:495-7.

13 Servant G, Nicks JJ, Donn SM, Bandy KP, Lathrop C, Dechert RE, et al. Feasibility of applying flowvynchronized ventilation to very low birthweight infants. synchronized ventilation to very
Respiratory Care 1992; 37:249-53.

14 Gerhardt TO. Measurement of pulmonary mechanics in the NICU: limitations to its usefulness. Neonatal Respiratory Diseases 1995; 5:1-10. 